Arch. Tierz., Dummerstorf 43 (2000) 3, 213-222 Aus dem Institut für Tierzucht und Vererbungsforschung', dem Institut für Pathologie ${ }^{2}$ und der Klinik für
Rinderkrankheiten ${ }^{3}$ der Tierărztlichen Hochschule Hannover

CORD DRÖGEMÜLLER', STEFAN NEANDER', HEIKE KLIPPERT' ${ }^{\prime}$, HEIDI KUIPER', LARS KUTSCHKE', SILVIA GUIONAUD ${ }^{2}$, SIEGFRIED UEBERSCHÄR' ${ }^{2}$, HENNER SCHOLZ ${ }^{3}$ und OTTMAR DISTL ${ }^{1}$

\title{
Genetische Analyse der kongenitalen Hypotrichose mit Anodontie beim Rind
}

Herrn Professor Dr. D. Simon zum 70. Geburtstag gewidmet

\section{Summary}

Title of the paper: Genetic analysis of congenital hypotrichosis with anodontia in cattle

In three male German Holstein calves, black and white spotted, congenital hypotrichosis and nearly complete anodontia was observed. The pedigree of the probands being maternal halfsibs and grandmaternal grandsons supports a monogenic, X-linked recessive inheritance. Chromosomal Xq-deletions were not detected. Similar congenital anomalies are known in tabby mice and in humans as anhidrotic ectodermal dysplasia (ED1, EDA). The human gene causative for ED1 and the Tabby gene are located on the X-chromosome. Therefore, the moleculargenetic marker analysis in our material was restricted on the X-chromosome. Using identical by descend mapping based on 20 microsatellites, we were able to identify an exclusively joint genomic region on the long arm of the X-chromosome among the affected calves. The putative location of the gene locus for congenital hypotrichosis with anodontia maps into the region of the marker BM4604 on position $76.5 \mathrm{cM}$ of the X-chromosome.

Key Words: cattle, hypotrichosis, anodontia, genetics

\section{Zusammenfassung}

Bei drei männlichen Kälbern der Rasse Deutsche Holsteins, Farbrichtung Schwarzbunt, wurde kongenitale Hypotrichose mit fast vollständiger Anodontie (angeborener verminderter Haarwuchs und fast vollständiges Fehlen der Zähne) beobachtet. Das Pedigree der Probanden, die mütterliche Halbgeschwister sind und auf eine gemeinsame mütterliche Großmutter zurückgehen, spricht für eine monogen, X-chromosomal rezessive Vererbung. Deletionen am X-Chromosom waren nicht nachzuweisen. Ähnliche kongenitale Anomalien treten bei Tabby-Mäusen und beim Menschen als ektodermale anhidrotische Dysplasie (ED1, EDA) auf. Das dafür verantwortliche Gen ist auf dem X-Chromosom lokalisiert. Deshalb wurde die molekulargenetische Markeranalyse in diesem Material auf das X-Chromosom begrenzt. Mittels 20 Mikrosatelliten-Markern ließ sich eine ausschließlich für die betroffenen Kälber gemeinsame Genomregion auf dem langen Arm des XChromosoms nachweisen. Demnach befindet sich der Genort für kongenitale Hypotrichose mit Anodontie im Bereich des Markers BM4604 auf der Position 76,5 cM des X-Chromosoms.

Schlüsselwörter: Rind, Hypotrichose, Anodontie, Genetik

\section{Einleitung}

Beim Rind sind gemeinsame Entwicklungsstörungen von Haaren, Schweißdrüsen und Zähnen sehr selten. Die kongenitale Hypotrichosis in Verbindung mit Ano- oder Oligodontie ist bisher nur in Einzelfällen bekannt geworden (STÖBER et al., 1995). Entsprechend dem verschieden stark ausgeprägten Fehlen der Zahnanlagen werden 
folgende zwei Erscheinungsformen beim Rind unterschieden:

- kongenitale Hypotrichose mit vollständiger oder partieller Anodontie (HAD: Hypotrichose-Anodontie-Defekt)

- kongenitale Hypotrichose mit partiellem oder völligem Fehlen der Incisivi (HID: Hypotrichose-Incisivi-Defekt)

Beim Rind wurden für HAD Einzelfälle von DRIEUX et al. (1950), RIECK (1985) und BRAUN et al. (1988) bzw. ANSARI et al. (1987) beschrieben. Außer beim Rind wurde HAD auch beim Hund beobachtet. CASAL et al. (1997) wiesen einen monogenen X-gekoppelten rezessiven Erbgang nach. DRIEUX et al. (1950) berichteten über drei Bullenkälber, die von einer Normänner x Maine-Anjou Kreuzungskuh abstammten. Diese Kälber wiesen eine Haarlosigkeit über den ganzen Körper, eine komplette Anodontie und Makroglossie auf. Für diese Fälle erschien ein X-gekoppelter, monogen rezessiver Erbgang als die plausibelste Erklärung. RIECK (1985) erwähnte einen Fall eines Schwarzbunten Bullenkalbes mit 50\% US-Holstein Friesian Anteil mit Hypotrichose und gleichzeitigem Fehlen der Incisivi, der Prämolaren und des ersten Molaren. Ähnliche Anomalien wies der bisher einzige Fall eines weiblichen Tieres auf. Ein Schweizer Fleckvieh x Red Holstein Rind fiel durch Hypotrichose und unvollständige Ausbildung der Schneide- und Backenzähne auf (ANSARI et al., 1987; BRAUN et al., 1988). Jedoch passt der aus der Schweiz stammende Fall aufgrund des Pedigrees nicht zu einem X-gekoppelten Erbgang. Die Expression des Defekts wurde deshalb mit einer Deletion im langen Arm des XChromosoms und einer Inaktivierung des zweiten X-Chromosoms erklärt.

Die zweite Form (HID) wurde von COLE (1919) und WIJERATNE et al. (1988) beschrieben. Die von COLE (1919) beschriebenen fünf Fälle gehen auf einen normal behaarten Bullen zurück, dem die Schneidezähne zum Teil fehlten oder nur sehr klein ausgebildet waren. Die Minderbehaarung zeigte sich vor allem an Kopf und Nacken, verschwand aber im Laufe der Entwicklung bei allen fünf Kälbern. Ob der Bulle ebenfalls diese Art von Hypotrichose als Kalb aufwies, konnte nicht geklärt werden. Drei von den fünf Kälbern wiesen ähnliche Zahnfehler wie ihr Vater an den Incisivi auf. Die übrigen 15 bis 20 Nachkommen des Bullen in derselben Herde waren unauffällig hinsichtlich Behaarung und Zahnausbildung. Eine erbliche Ursache wurde vermutet. WIJERATNE et al. (1988) beschrieben vier befallene Bullenkälber mit Hypotrichose und völligem Fehlen der Incisivi. Die beiden Mütter und die gemeinsame Großmutter wiesen kurzes, glanzloses Haar und grau statt schwarz pigmentierte Haut auf. Die Autoren schlossen auf eine genetische Ursache mit einem X-gekoppelten, monogen unvollständig dominanten Erbgang. Da das Defektallel bei den heterozygoten weiblichen Tieren nur geringe Veränderungen in der Behaarung verursachte, plädierten die Autoren für einen unvollständig dominanten Erbgang. Die vollständige Expression des Defekts verhielt sich wie bei HAD X-gekoppelt, monogen rezessiv.

Beim Menschen stellt die anhidrotische ektodermale Dysplasie (ED1, auch EDA, EDA1, XLHED oder Christ-Siemens-Touraine-Syndrom, CST) das zu HAD fast identische Krankheitsbild dar. Der Erbgang ist X-gekoppelt, monogen rezessiv. Bei 
Anlageträgerinnen fällt eine Reduktion der Anzahl, Größe und des Ausreifungsgrades der Schweiss- und Talgdrüsen sowie der Haarfollikel auf. Unter der Vielzahl von ektodermalen Dysplasien ist ED1 beim Menschen relativ häufig. Der Genort für ED1 wurde beim Menschen auf dem X-Chromosom in der Region Xq12-q13.1 lokalisiert (THOMAS et al., 1993). Das Genprodukt ist ein kleines Transmembranprotein mit einer extrazellulären Kollagendomäne (Ektodysplasin 1 oder Ektodysplasin A1). Es werden die Isoformen I und II unterschieden, wobei bei jeder Isoform wiederum verschiedene allelische Varianten auftreten können. ED1-Patienten fallen durch Genmutationen auf, in deren Folge Genruptionen und gestörte Proteinfunktion auftreten. Der erste Bericht über Mutationen in dem ED1-Gen beim Menschen stammt von KERE et al. (1996). In der Folgezeit wurde eine Vielzahl von Mutationen in dem ED1-Gen entdeckt (FERGUSON et al., 1998; MONREAL et al., 1998; BAYES et al., 1998). Eine genetische Heterogenität, d.h. daß andere Genorte dasselbe Krankheitsbild hervorrufen, ist mit hoher Wahrscheinlichkeit beim Menschen auszuschließen. Der Genort für ED1 weist zum Tabby-Genort der Maus (Ta) eine hohe Homologie auf (FERGUSON et al., 1997; SRIVASTAVA et al., 1997). Der Tabby-Genort ist ebenfalls auf dem X-Chromosom an der Position 37,0 cM lokalisiert. Die Homologie zwischen dem humanen und murinen Protein ist sehr hoch. Tabby-Mäuse haben ein dünnes, seidenartiges Fell bei Fehlen der Schweissdrüsen und der Hautfurchen an den Pfoten. Die Zähne fehlen oder sind mißgestaltet und die Öffnung der Lidspalten erfolgt nach der Geburt erst mit Verzögerung.

In der vorliegenden Arbeit sollen drei Fälle von männlichen Kälbern der Rasse Deutsche Holsteins, Farbrichtung Schwarzbunt, vorgestellt werden, bei denen Hypotrichose mit fast vollständiger Anodontie (HAD) diagnostiziert wurde.

2. Material und Methoden

Die drei männlichen Probanden der Rasse Deutsche Holsteins, Farbrichtung Schwarzbunt, $(\mathrm{DH}, \mathrm{S})$ stammen von einem der Milchleistungsprüfung angeschlossenem Betrieb. Die Tiere fielen bei der Geburt durch ihre weitgehende Haarlosigkeit auf. Das erste befallene Bullenkalb (Ziki) wurde bis zu einem Alter von 3 Monaten aufgezogen und dann geschlachtet. Das zweite Tier (Zonas) wurde vom Institut für Tierzucht und Vererbungsforschung der Tierärztlichen Hochschule Hannover bis zu einem Alter von etwa 2 Jahren aufgezogen. Der dritte Proband (Zephir) hat jetzt ein Alter von 2,5 Monaten und befindet sich am o.g. Institut. Neben der klinischen Untersuchung der Tiere wurde eine Untersuchung der Haut von dem ersten betroffenen Tier am Institut für Tierpathologie der Ludwig-Maximilians-Universität München (Vorstand: Prof. Dr. W. Hermanns) und von den beiden weiteren Fällen am Institut für Pathologie der Tierärztlichen Hochschule Hannover durchgeführt. Nach Ermittlung des Pedigrees wurde eine Untersuchung von Mikrosatelliten auf dem XChromosom durchgeführt (Abb.). Bei zwei betroffenen Bullen (Zonas und Zephir), deren Mutter Zilli und weiblichen Verwandten erfolgte eine zytogenetische Untersuchung. 


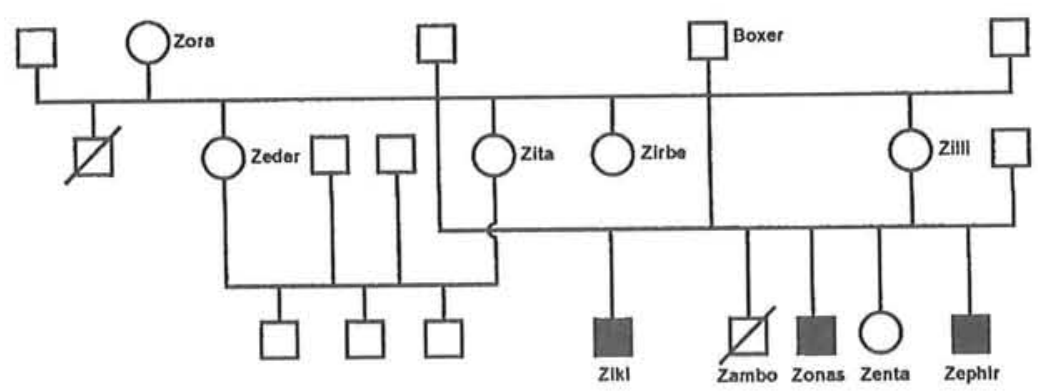

Abb.: Pedigree für die von kongenitaler Hypotrichose mit fast vollständiger Anodontie betroffenen Kälber der Rasse Deutsche Holsteins (Pedigree for the German Holstein calves affected by congenital hypotrichosis and nearly complete anodontia)

Für die Chromosomenanalyse wurde eine sterile Blutprobe aus der Vena jugularis entnommen. Die zytogenetische Untersuchung erfolgte aus Lymphozytenkulturen. Dazu wurden heparinisierte Vollblutproben mit Kulturmedium, antibiotischem Zusatz und Phytohämagglutinin als Zellmitogen versetzt und anschließend für 72 Stunden bei $38^{\circ} \mathrm{C}$ und $5 \% \quad \mathrm{CO}_{2}$-Begasung inkubiert. Die Zellkultur wurde mit Colcemid beendet. Ein Zellsediment wurde durch anschließende Zentrifugation und eine hypotone Behandlung mit 0,075 M KCl-Lösung gewonnen. Nach dreimaliger Aufnahme des Zellsediments in die Fixierlösung (1 Teil Essigsäure, 3 Teile Methanol) und jeweils anschließender Zentrifugation wurde die Zellsuspension in eine Pipette aufgenommen und auf einen Objektträger aufgetropft. Die Darstellung der Chromosomen in der Metaphase erfolgte mittels Giemsafärbung oder GTG-Bänderung. Die Aufnahmen der Metaphasen wurden mit einer CCD-Kamera und der Software IPLab 2.3 erstellt.

Für die histologische Untersuchung wurden Hautproben bei den ersten beiden Fällen (Ziki und Zonas) vorwiegend vom Kopf (Stirn, Augenbraue, Flotzmaul, Ohr, Augenlid), Hals, Bauch und Rücken entnommen. Bei dem noch lebenden Tier (Zephir) wurden Stanzproben im Alter von 6 Wochen von Wange, Hals, Ohr und Unterbrust mit $6 \mathrm{~mm}$ Dicke entnommen. Alle Proben wurden in $10 \%$-igem Formalin fixiert und in Paraffin eingebettet. Von den Hautproben wurden Serienschnitte gefertigt. Die histologischen Schnitte wiesen eine Dicke von $3 \mu \mathrm{m}$ auf und wurden mit Hämalaun-Eosin (HE) angefärbt.

Insgesamt wurden 20 Mikrosatelliten, die auf dem X-Chromosom des Rindes lokalisiert sind, bei zehn Tieren genotypisiert. Die Auswahl der Mikrosatelliten erfolgte nach der genetischen Karte des USDA, Clay Center, Nebraska, USA

(http://sol.marc.usda.gov/cgi-bin/species_chromosome?cattle+xy). Zehn der ausgewählten Mikrosatelliten sind über das gesamte X-Chromosom einschließlich der pseudoautosomalen Region verteilt und decken mit einem Abstand von ca. 15 cM einen Bereich von 143,9 cM auf der genetischen Karte vom X-Chromosom des Rindes ab (SONSTEGARD et al., 1997). Aufgrund der vollständigen Konservierung des X-Chromosoms zwischen Mensch und Rind (SOLINAS-TOLDO et al., 1995) 
und der physikalischen Lokalisation des ED1-Gens auf Xq12-q13.1 beim Menschen (THOMAS et al., 1993) wurden zusätzlich zehn weitere Mikrosatelliten aus den Abschnitten zwischen $60 \mathrm{cM}$ und $80 \mathrm{cM}$ des X-Chromosoms der bovinen Genkarte in die Untersuchung miteinbezogen (Tab. 1). Die Isolierung der genomischen DNA aus EDTA-Blut erfolgte mit dem Puregene Genomic DNA Isolation Kit (Fa. Gentra, Minneapolis, USA). Für die Amplifizierung der Mikrosatelliten mittels PCR betrug das Reaktionsvolumen $15 \mu \mathrm{l}$. Es enthielt ca. $25 \mathrm{ng}$ genomische DNA, 1x PCRPuffer, 1,5 mM $\mathrm{MgCl}_{2}$, je 7,5 pmol Primer, $100 \mathrm{mM}$ je Desoxyribonucleosidtriphophat (dNTP) und 1 U Taq DNA Polymerase (Fa. Promega, Mannheim). Eine Auftrennung der PCR-Amplifikate erfolgte für neun Marker auf denaturierenden 6\%-Polyacrylamidgelen und anschließender Silberfärbung. Für die übrigen elf Marker wurden fluoreszenzmarkierte Primer in der PCR eingesetzt, wonach die PCR-Produkte auf einem automatischen Sequenziersystem (LI-COR 4200S-2, Fa. MWG Biotech, Ebersberg) analysiert wurden.

\section{Ergebnisse und Diskussion}

\section{Befunde an den betroffenen Kälbern}

Beim ersten Fall war das Kalb (Ziki) bei der Geburt am ganzen Körper haarlos. Im Lauf der weiteren Entwicklung zeigte sich ein feiner Flaum von Haaren und eine stärkere Behaarung konnte nur an den Hinterbeinen, der Flanke, dem Bauch, den Vordergliedmaßen bis zu der Höhe des Ellbogengelenks und am Triel festgestellt werden. Die Schwanzquaste war nur zu etwa 1/3 der üblichen Länge ausgebildet. In der Maulhöhle konnte nur je ein Backenzahn am rechten und linken Oberkiefer palpiert werden. Die Haut wies v.a. an Kopf und Hals eine feine Fältelung auf. Aufgrund der geringen Behaarung und der feinen Haut war das Kalb an den exponierten Körperstellen sehr anfällig für Hautverletzungen. Eine Rauhfutter- und Grasaufnahme war nicht möglich. Klauen- und Hodenentwicklung waren ohne auffällige Befunde. Im Oberkiefer waren beiderseits je zwei Backenzähne im Bereich der Molaren angelegt; je einer war durch den Knochen und das Zahnfleisch durchgebrochen, die beiden anderen Backenzähne waren nur durch den Knochen durchgebrochen, aber nicht durch das Zahnfleisch. Im Unterkiefer konnten beiderseits die Zahnanlagen von je zwei Molaren festgestellt werden. Die histopathologischen Untersuchungen von Hautproben von Kopf und Rücken zeigten nur einige spärlich entwickelte Haare mit wenig differenzierter Haarwurzel sowie dilatierte Schweißdrüsen.

Der zweite Fall (Zonas) ist ein mütterlicher Halbbruder zu Ziki. Die kongenitale Hypotrichose war nicht so deutlich ausgeprägt wie beim ersten Fall und vorwiegend auf den Kopf sowie die angrenzende Halspartie beschränkt. Die Zahnbefunde auf dem Röntgenbild zeigten ebenfalls eine fast vollständige Anodontie. Es konnten im linken Oberkiefer zwei Zahnanlagen und im rechten Oberkiefer drei Zahnanlagen für Backenzähne festgestellt werden. Im Unterkiefer befanden sich links eine und rechts zwei Zahnanlagen für Backenzähne. Der Zahnbogen im Oberkiefer war deutlich verkürzt und konvex geformt. Histologisch konnte eine verringerte Anzahl von 
Haarfollikeln und Schweissdrüsen nachgewiesen werden. An der Ohraußenseite fehlten Haare und Schweissdrüsen völlig. Die seromucösen Nasolabialdrüsen konnten nicht nachgewiesen werden. Horn- und Klauenentwicklung waren ohne besonderen Befund. Der Bulle entwickelte sich gut, auch wenn er die Gewichtszunahme von Mastbullen nicht erreichte. Eine Wachstumsbeeinträchtigung und eine Störung der Pansenfunktion konnte durch eine Futterration aus Cobs und pelletiertem Kraftfutter vermieden werden.

Der dritte Fall (Zephir) ist ebenso ein mütterlicher Halbbruder zu Ziki. Das Erscheinungsbild der kongenitalen Hypotrichose ähnelte dem vorhergehenden Fall sehr deutlich. Die Hypotrichose war vorwiegend auf den Kopf sowie die angrenzende Halspartie beschränkt. Die Zahnbefunde wiesen ebenfalls eine fast vollständige Anodontie auf. Es konnte beiderseits nur je ein Backenzahn im Oberkiefer palpiert werden. Histologisch bot sich dasselbe Bild wie bei dem vorhergehenden Fall Zonas.

\section{Erbgangsanalyse}

Das Pedigree läßt sich mit einem X-gekoppelten, monogen rezessiven Erbgang erklären (Abb.). Ein autosomaler, monogen rezessiver Erbgang erscheint sehr unwahrscheinlich, da bei einem so seltenen Auftreten dieses Defekts die Allelfrequenz sehr niedrig ist und deshalb das Zusammentreffen von drei heterozygoten Bullen mit unterschiedlicher Abstammung in einem Betrieb nahezu ausgeschlossen werden kann. Eine zytoplasmatische Vererbung kommt ebenfalls als Erklärungshypothese nicht in Betracht, da nur die männlichen Nachkommen betroffen waren. In dem vorliegenden Pedigree kommt Zora als Urspung für das Defektallel auf dem X-Chromosom in Frage. Die Bullen können keine Träger des Defektallels sein, da sie phänotypisch unauffällig waren. Das Gründertier Zora hat wahrscheinlich das Defektallel an Zilli und diese an die betroffenen Bullenkälber Ziki, Zonas und Zephir übertragen. Die zu Zilli mütterlichen Halbschwestern Zeder, Zita und Zirbe sind mit einer Wahrscheinlichkeit von $50 \%$ Anlageträgerinnen, was anhand des Pedigrees noch nicht verifiziert werden konnte.

\section{Molekular- und zytogenetische Analyse}

Die Karyogramme von Zonas $(2 n=60, X Y)$, Zephir $(2 n=60, X Y)$, Zilli $(2 n=60, X X)$ und Zenta $(2 n=60, X X)$ zeigten keine Befunde, die auf lichtmikroskopisch erkennbare numerische oder strukturelle Anomalien der Chromosomen hinwiesen.

In der molekulargenetischen Mikrosatellitenmarkeranalyse wurde nur das XChromosom berücksichtigt, da die Fälle von DRIEUX et al. (1950) und das vorliegende Pedigree für eine X-gekoppelte Vererbung sprechen und aufgrund der vergleichenden Genomkartierung eine vollständige Konservierung des X-Chromosoms zwischen Mensch und Rind nachgewiesen wurde (SOLINAS-TOLDO et al., 1995). Die Markeranalyse basiert auf dem Vergleich von abstammungsgleichen Allelen zwischen den mit HAD befallenen Kälbern und ihren Vorfahren. Nur die Genomregionen können als Lokalisation für den Genort HAD in Frage kommen, die einen gemeinsamen Ursprung bei der mütterlichen Großmutter Zora haben und auf die 
Befallenen übertragen wurden. Die Region zwischen den Markern BM6017 und XBM111 im Bereich von $4,7 \mathrm{cM}$ bis $60,4 \mathrm{cM}$ sowie die Region um den Marker BMS417 bei $69,9 \mathrm{cM}$ und distal von dem Marker BMC6021 bei 91,3 cM scheiden als mögliche Lokalisationen für den Genort HAD auf dem X-Chromosom aus, da die befallenen Kälber unterschiedliche Haplotypen von der Mutter erhielten. Nicht informativ waren die Marker BMS1820, BMS485, RM350, BMS807 und ILSTS017 für die Nachkommen von Zilli (Tab. 1). Die gemeinsam an die betroffenen Halbbrüder weitergegebenen Chromosomenabschnitte können über die sieben abstammungsgleichen Allele BMS513, XBM19, BM4604, BMS2592, XBM84, BMS500, BMC6021 auf dem X-Chromosom identifiziert werden (Tab. 2).

\section{Tabelle 1}

$\mathrm{X}$-chromosomale Markergenotypen in dem untersuchten Pedigree (X chromosomal marker genotypes in the pedigree analysed)

\begin{tabular}{|c|c|c|c|c|c|c|c|c|c|c|c|}
\hline Marker & $\begin{array}{c}\text { Position } \\
\text { (cM) }\end{array}$ & Zora & Zilli & Ziki & Zonas & Zephir & Zenta & Zeder & Zita & Zirbe & Boxer \\
\hline BM6017 & 4,7 & $4 / 1$ & $2 / 1$ & $-/ 2$ & -11 & -12 & $3 / 2$ & $2 / 1$ & $3 / 4$ & $3 / 4$ & -13 \\
\hline BMS903 & 12,1 & $2 / 2$ & $3 / 2$ & $-/ 3$ & -12 & $-/ 3$ & $1 / 3$ & $4 / 2$ & $1 / 2$ & $1 / 2$ & $-/ 1$ \\
\hline HUJ121 & 40,0 & $1 / 2$ & $1 / 2$ & $-/ 1$ & -12 & $-/ 1$ & $1 / 1$ & $3 / 2$ & $1 / 2$ & $1 / 2$ & $-/ 1$ \\
\hline XBM111 & 60,4 & $2 / 2$ & $1 / 2$ & $-/ 1$ & -12 & -12 & $1 / 1$ & $1 / 2$ & $1 / 2$ & $1 / 2$ & $-/ 1$ \\
\hline BMS1820 & 69,8 & $2 / 1$ & $1 / 1$ & $-/ 1$ & -11 & 11 & $1 / 1$ & $2 / 1$ & $1 / 1$ & $1 / 1$ & -11 \\
\hline BMS417 & 69,9 & $2 / 3$ & $1 / 3$ & $-/ 1$ & -13 & -13 & $1 / 1$ & $2 / 3$ & $1 / 3$ & $1 / 3$ & $-/ 1$ \\
\hline BMS485 & 70,7 & $2 / 1$ & $1 / 1$ & $-/ 1$ & -11 & -11 & $1 / 1$ & $1 / 1$ & $1 / 1$ & $1 / 1$ & $-/ 1$ \\
\hline RM350 & 71,7 & $1 / 1$ & $1 / 1$ & $-/ 1$ & -1 & -11 & $1 / 1$ & $2 / 1$ & $1 / 1$ & $1 / 1$ & -11 \\
\hline BMS807 & 72,5 & $1 / 1$ & $1 / 1$ & $-/ 1$ & -11 & -11 & $1 / 1$ & $1 / 1$ & $1 / 1$ & $1 / 1$ & $-/ 1$ \\
\hline BMS513 & 73,5 & $2 / 1$ & $2 / 1$ & -11 & -11 & -11 & $1 / 2$ & $3 / 1$ & $1 / 1$ & $1 / 2$ & -11 \\
\hline XBM19 & 73,5 & $1 / 2$ & $1 / 2$ & -12 & -12 & -12 & $2 / 1$ & $1 / 2$ & $2 / 2$ & $2 / 1$ & $-/ 2$ \\
\hline ILSTS017 & 73,5 & $1 / 1$ & $1 / 1$ & -11 & -11 & -11 & $1 / 1$ & $1 / 1$ & $1 / 1$ & $1 / 1$ & -11 \\
\hline BM4606 & 76,5 & $2 / 1$ & $2 / 1$ & -11 & -11 & -11 & $2 / 2$ & $2 / 1$ & $2 / 1$ & $2 / 1$ & -12 \\
\hline BMS2592 & 80,0 & $1 / 1$ & $1 / 1$ & -11 & -11 & -11 & $2 / 1$ & $1 / 1$ & $2 / 1$ & $2 / 1$ & -12 \\
\hline XBM84 & 80,0 & $1 / 3$ & $3 / 3$ & -13 & -13 & -13 & $3 / 3$ & $2 / 3$ & $3 / 3$ & $3 / 1$ & -13 \\
\hline BMS500 & 80,9 & $1 / 2$ & $2 / 2$ & -12 & -12 & -12 & $3 / 2$ & $1 / 2$ & $3 / 2$ & $3 / 1$ & $-/ 3$ \\
\hline BMC 6021 & 91,3 & $2 / 2$ & $3 / 2$ & -12 & -12 & -12 & $1 / 3$ & $1 / 2$ & $1 / 2$ & $1 / 2$ & $-/ 1$ \\
\hline BMS2798 & 104,3 & $1 / 2$ & $3 / 2$ & -12 & -12 & $-/ 3$ & $2 / 3$ & $3 / 2$ & $2 / 2$ & $2 / 1$ & -12 \\
\hline XBM16 & 119,5 & $2 / 2$ & $1 / 2$ & -12 & -12 & $-/ 1$ & $3 / 1$ & $2 / 2$ & $3 / 2$ & $3 / 2$ & -13 \\
\hline TGLA325 & 143,9 & $3 / 1$ & $2 / 1$ & $3 / 1$ & $3 / 1$ & $1 / 2$ & $4 / 2$ & $4 / 1$ & $4 / 1$ & $4 / 1$ & $3 / 4$ \\
\hline
\end{tabular}

Der Marker ILSTS017 ist nicht informativ, jedoch die Wahrscheinlichkeit für eine Doppelrekombination ist sehr gering. Damit kann die Kandidatenregion auf den distalen Bereich des X-Chromosoms zwischen den Markern BMS513 und BMC6021 mit einer Ausdehnung von 17,8 cM eingeengt werden. Dieser Genabschnitt entspricht nach den komparativen Genkarten zwischen Mensch und Rind der erwarteten Region für die Lokalisation des ED1 Gens. Damit erweist sich das ED1 Gen als hochwahrscheinlicher Kandidatengenort für HAD beim Rind. Bei Ziki und Zephir 
Tabelle 2

Markeranalyse mittels Mikrosatelliten für das bovine X-Chromosom (Marker analysis using microsatellites of the bovine $\mathrm{X}$ chromosome)

\begin{tabular}{|c|c|c|c|c|c|c|}
\hline \multirow[t]{2}{*}{ Marker } & \multirow[t]{2}{*}{$\begin{array}{l}\text { Position } \\
\left(\mathrm{cM}^{1}\right)\end{array}$} & \multirow{2}{*}{$\begin{array}{c}\text { abstammungs- oder } \\
\text { statusgleiches Allel } \\
\text { bei Kälbern mit } \\
\text { HAD }\end{array}$} & \multicolumn{3}{|c|}{$\begin{array}{c}\text { abstammungs-/statusgleiches } \\
\text { Allel } \\
\text { heterozygot bei }\end{array}$} & \multirow{2}{*}{$\begin{array}{c}\text { status- } \\
\text { gleiches } \\
\text { Allel beim Bullen } \\
\text { Boxer }\end{array}$} \\
\hline & & & Zora & Zilli & Zita & \\
\hline BMS513 & 73,5 & 1 & ja & ja & nein & ja \\
\hline XBM19 & 73,5 & 2 & ja & ja & nein & ja \\
\hline ILSTSO17 & 73,5 & 1 & nein & nein & nein & ja \\
\hline BM4604 & 76,5 & 1 & ja & ja & ja & nein \\
\hline BMS2592 & 80,0 & 1 & nein & nein & ja & nein \\
\hline XBM84 & 80,0 & 3 & ja & nein & nein & ja \\
\hline BMS500 & 80,9 & 2 & ja & nein & ja & nein \\
\hline BMC6021 & 91,3 & 2 & nein & ja & ja & nein \\
\hline
\end{tabular}

wurde jeweils dieses chromosomale Segment durch eine Rekombination zwischen den Chromatiden übertragen, während bei Zonas ein Crossing over nicht nachweisbar war. Vor allem die Region um den Mikrosatelliten BM4606 kommt als mögliche Lokalisation für das ED1 Gen in Frage. Allein die Allele des Markers BM4604 verhalten sich wie bei einem X-gekoppelten rezessiven Erbgang. Das Markerallel "1" nimmt seinen Ursprung bei der mütterlichen Großmutter Zora und wird über Zilli zu den betroffenen Bullenkälbern Ziki, Zonas und Zephir übertragen. Alle weiblichen Verwandten der Betroffenen sind für das Markerallel heterozygot, und der Bulle Boxer trägt ein anderes Allel. Die Markeranalyse in Verbindung mit der populationsgenetischen Studie und der vergleichenden Genomkartierung gibt damit einen Hinweis für die Lage des ED1 Gens in der Region um den Marker BM4604.

\section{Danksagung}

Die Autoren bedanken sich sehr herzlich bei Herrn Dr. Gauchel, da er uns über diese Fälle benachrichtigt hat. Herrn Prof. Dr. W. Hermanns, Institut für Tierpathologie der Ludwig-Maximilians-Universität München, sei für die pathohistologische Untersuchung eines Kalbes recht herzlich gedankt.

\section{Literatur}

ANSARI, H.A.; HEDIGER, R.; BRAUN, U.; SÜSS, U.; STRANZINGER, G.:

A case of partial Xq-deletion in a viable hairless calf. Ann. Meet. Amer. Soc. Animal Sci. (Abstr.) 79 (1987), 193

BAYES, M.; HARTUNG, A.J.; EZER, S.; PISPA, J.; THESLEFF, I.; SRIVASTAVA, A.K.; KERE, J.:

The anhidrotic ectodermal dysplasia gene (EDA) undergoes alternative splicing and encodes ectodysplasin-A with deletion mutations in collagenous repeats. Hum. Mol. Genet. 7 (1998), 16611669

BRAUN, U.; ANSARI, H.A.; HEDIGER, R.; SÜSS, U.; EHRENSPERGER, F.:

Hypotrichose und Oligodontie, verbunden mit einer Xq-Deletion, bei einem Kalb der schweizerischen Fleckviehrasse. Tierärztl. Praxis 16 (1988), 39-44 
Arch. Tierz. $43(2000) 3$

CASAL, M.L.; JEZYK, P.F.; GREEK, J.M.; GOLDSCHMIDT, M.H.; PATTERSON, D.F.: COLE, L.J.: $\mathrm{X}$-linked ectodermal dysplasia in the dog. J. Hered. 88 (1997), 513-516

A defect of hair and teeth in cattle - probably hereditary. J. Heredity 10 (1919), 303-306

DRIEUX, H.; PRIOUZEAU, M.; THIÉRY, G.; PRIOUZEAU, M.-L.: Hypotrichose congénitale avec anodontie, acérie et macroglossie chez le veau. Rec. Méd. Vét. 126 (1950), 385-399

FERGUSON, B.M.; BROCKDORFF, N.; FORMSTONE, E.; NGYUEN, T.; KRONMILLER, J.E.; ZONANA, J.:

Cloning of Tabby, the murine homolog of the human EDA gene: evidence for a membrane-associated protein with a short collagenous domain. Hum. Molec. Genet. 6 (1997), 1589-1594

FERGUSON, B.M.; THOMAS, N.S.T.; MUNOZ, F.; MORGAN, D.; CLARKE, A.; ZONANA, J.: Scarcity of mutations detected in families with X-linked hypohidrotic ectodermal dysplasia: diagnostic implications. J. Med. Genet. 35 (1998), 112-115

KERE J.; SRIVASTAVA, A.K.; STONEN, A.; ZONANA, J.; THOMAS, N.; FERGUSON, B.; MUNOZ, F.; MORGAN, D.; CLARKE, A.; BAYBAYAN, P.; CHEN, E.J.; EZER, S.; SAARIALHO-KERE, U.; DE LA CHAPELLE, A.; SCHLESSINGER, D.:

$\mathrm{X}$-linked anhidrotic (hypohidrotic) ectodermal dysplasia is caused by mutation in a novel transmembrane protein. Nature Genetics 13 (1996), 409-416

MONREAL, A.; ZONANA, J.; FERGUSON, B.:

Identification of a new splice form of the EDA1 gene permits detection of nearly all X-linked hypohidrotic ectodermal dysplasia mutations. Am. J. Hum. Genet. 63 (1998), 380-389

RIECK, G.W.:

Hypotrichie - Hypodontie - Syndrom beim Rind. Dtsch. Tierärzl. Wschr. 92 (1985), 328-329

SOLINAS-TOLDO, S.; LENGAUER, C.; FRIES, R.:

Comparative Genome Map of Human and Cattle. Genomics 27 (1995), 489-496

SONSTEGARD T.S.; LOPEZ-CORRALES, N.L.; KAPPES, S.M.; STONE, R.T.; AMBADY, S.; PONCE DE LEON, F.A.; BEATTIE, U.C.W.:

An integrated genetic and physical map of the bovine X Chromosome. Mammalian Genome 8 (1997), $16-20$

SRIVASTAVA A.K.; PISPA, J.; HARTUNG, A.J.; DU, Y.; EZER, S.; JENKS, T.; SHIMADA, T.; PEKKANEN, M.; MIKKOLA, M.L.; KO, M.S.A.; THESLEFF, I.; KERE, J.; SCHLESSINGER, D.: The Tabby phenotype is caused by mutation in a mouse homologue of the EDA gene that reveals novel mouse and human exons and encodes a protein (ectodysplasin-A) with collagenous domains. Proc. Nat. Acad. Sci. 94 (1997), 13069-13074

STÖBER, M.; WEITZE, K.-F.; HOEDEMAKER, M.; POHLENZ, J.; LIEBLER, E.; WURM, S.; HARLIZIUS, B.; TREVIRANUS, A.; SISSKO, J.:

Ausgebreitete Minderbehaarung mit horizontalem Vorbiß der Schneidezähne und unterentwickeltem Genitale, eine angeborene Mißbildung beim deutschen Schwarzbunten Rind. Tierärztliche Umschau 50 (1995), 224-239

THOMAS, N.S.T.; CHELLY, J.; ZONANA, J.; DAVIES, K.J.P.; MORGAN, S.; GAULT, J.; RACH, K.A.; BUCHLE, V.J.; BROCKDORFF, N.; CLARKE, A.; MONACO, A.:

Characterisation of molecular DNA rearrangements within the Xq12-q13.1 region, in three patients with X-linked hypohidrotic ectodermal dysplasia (EDA). Hum. Molec. Genet. 2 (1993), 1679-1685

WIJERATNE, W.V.S.; O'TOOLE, D.; WOOD, L.; HARKNESS, J.W.:

A genetical, pathological and virological study of congenital hypotrichosis and incisor anodontia in cattle. Vet. Record 122 (1988), 149-152

Eingegangen: 07.03.2000

Alzeptiert: 11.04 .2000 
Anschriften der Verfasser

Dr. CORD DRÖGEMÜLLER, STEFAN NEANDER, HEIKE KLIPPERT, HEIDI KUIPER, Dr. LARS KUTSCHKE, Prof. Dr. OTTMAR DISTL

Institut für Tierzucht und Vererbungsforschung der Tierärztlichen Hochschule Hannover Bünteweg 17p

D-30559 Hannover

E-Mail: cdroegem@zucht.tiho-hannover.de und odistl@zucht.tiho-hannover.de

Dr. SILVIA GUIONAUD, Prof. Dr. SIEGFRIED UEBERSCHÄR

Institut für Pathologie der Tierärztlichen Hochschule Hannover

Bünteweg 17

D-30559 Hannover

Prof. Dr. HENNER SCHOLZ

Klinik für Rinderkrankheiten der Tierärztlichen Hochschule Hannover

Bischofsholer Damm 15

D-30173 Hannover 